\title{
Conceptual design and RCS property research of three-surface strike fighter
}

\author{
Kuizhi Yue* \\ Department of Airborne Vehicle Engineering, Naval Aeronautical and Astronautical University, Yantai, 264001, China \\ School of Aeronautic Science and Engineering, Beijing University of Aeronautics and Astronautics, Beijing 100191, China
}

\section{Yifeng Tian** and Hu Liu**}

School of Aeronautic Science and Engineering, Beijing University of Aeronautics and Astronautics, Beijing 100191, China

\section{Wei Han**}

Department of Airborne Vehicle Engineering, Naval Aeronautical and Astronautical University, Yantai, 264001, China

\begin{abstract}
This paper mainly focuses on the conceptual design and stealth performance of the three-surface military aircraft. A threedimensional (3-D) digital mock-up of the three-surface strike fighter with stealth feature was designed and the schemes of carrying missiles were analyzed in CATIA. Based on physical optics principle and the Method of Equivalent Currents (MEC), a numerical simulation of the RCS feature of the aircraft was carried out with RCSPlus which is a software designed by Beihang University. The paper contributes to the RCS feature analysis of the whole plane and different parts on X-band, S-band and UHF-band and a comparison of RCS feature to Su-37 and T-50 military aircraft is drawn. On X-band, the pitch angle of the incident wave was $0^{\circ}$, and the result shows: (1) Compared with Su-37 aircraft, the forward scattering RCS of the three-surface strike aircraft was reduced to $14.9 \%$, the side scattering RCS to $9.6 \%$ and the back scattering RCS to $40.2 \%$. (2) Compared with T-50 aircraft, the forward scattering RCS was reduced to $38.61 \%$, and the side scattering RCS to $67.26 \%$. This paper should be useful for researchers in conceptual design and stealth technology of the military aircraft.
\end{abstract}

Key words: : stealth, conceptual design, strike fighter, numerical simulation.

\section{Introduction}

It is widely known that the four main aerodynamic configurations include the three-surface aerodynamic configuration, the normal configuration, the canard configuration and the flying-wing configuration. The aerodynamic lifting surface of the three-surface military aircraft is composed of canard, wing and horizontal tail. Although the canard adds weight to three-surface aircraft, it is widely used on military aircraft because of excellent maneuvering and aerodynamic performance. The three- surface configuration is utilized in a large amount of military aircrafts, such as Russian aircrafts Su-33 and Su-37, the American aircraft F-15S/MTD , the Japanese aircraft T-2CCV and so on. Su-33 is one of the main types of fighters in Russian naval aviation, which has served more than 20 years in aircraft carrier "Admiral Kuznetsov". The Chinese authorities also direct special attention to the three-surface aircraft research and have made some considerable achievements so far.

To meet evolving operational requirements, most modern advanced fighter planes such as American aircraft B-2, F-22 $2^{[1]}$, $\mathrm{F}-35^{[2]}, \mathrm{X}-45, \mathrm{X}-47$ involve stealth design. Domestic and foreign
This is an Open Access article distributed under the terms of the Creative Commons Attribution Non-Commercial License (http://creativecommons.org/licenses/by$\mathrm{nc} / 3.0 /$ which permits unrestricted non-commercial use, distribution, and reproduction in any medium, provided the original work is properly cited. (c) * ph. D, Corresponding author : yuekuizhi 2000@sohu.com ** ph. D 
experts have devoted a great deal of efforts to study stealth technology including the radar stealth ${ }^{[3]}$, the infrared stealth, the visible light stealth and the acoustic stealth. The design of stealthy fuselage, stealthy wings, stealthy air inlets, stealthy surface cracks $^{[4]}$ and some other stealth shape critical technology have been achieved. Additionally, different kinds of stealth materials and RCS algorithms have been obtained.

In reference [5], the numerical simulation of RCS comparison of the metal cone and the metal cone covering with plasma provides a reference algorithm for the RSC calculation. Reference [6] deals with the integration computing technology to identify the main scattering resource of complex targets. In reference [7], a dimension reduction model is built to simulate 3-D electromagnetic scattering and forecast the monostatic radar scattering cross section quickly. In order to obtain the RCS of the aircraft, a physical diffraction theory is proposed to calculate wedge diffraction in [8]. Reference [9] uses the finite difference time domain method to get the RCS of the aircraft via changing the 2-D scattering field to far field based on the equivalence principle. A research into the stealth performance of different radio frequency with different distance or different weather conditions is carried out in [10]. Reference [11] calculates the infrared radiation characteristic of the jet mixing enhancement by pulsed blowing in 3 to 5 microns bands and analyzes the infrared stealth properties. A comparison is made between the stealth properties of two aircrafts with missiles inside or outside in [12] and the results show that the aircraft with missiles inside has much better stealth properties. Reference [13] analyzes the stealth features of the fighter with double vertical tails outward and shows that double vertical tails outward have a great influence on the side scattering RCS of the fighter. Although enormous efforts have been taken to study different aspects of aircraft conceptual design and stealth technology, further research is still needed since there are few papers published on three-surface strike fighter with stealth feature.

This paper centralizes the target on the deficiency of the previous research. Based on CATIA, a 3-D digital mock-up of three-surface strike fighter is designed. And the numerical simulation of RCS performance of the three-surface aircraft with stealth feature has been obtained based on the principle of physical optics and the MEC with RCSPlus which is a software designed by Beihang University. Then the forward, the back and the side scattering RCS properties at different pitch angles are presented and a comparison of corresponding parameters to Su-37 and T-50 is made such that the stealth features of the conceptual design aircraft was obtained.

\section{Theoretical basis}

The theoretical basis to calculate the aircraft RCS includes two parts: the numerical simulation method and the experimental verification.

\subsection{Numerical simulation method}

On condition that the geometric shape of the aircraft is primarily considered without taking the stealth coating into account, the numerical simulation method to calculate the RCS is the method of physical optics and equivalent currents. The physical optics is used to calculate the facets scattering of the aircraft and the MEC is employed to calculate the edge diffraction ${ }^{[14,15]}$

The formula of the physical optics is shown as below ${ }^{[12,13]}$ :

$$
\sqrt{\sigma_{p o}}=-\mathrm{j} \frac{k}{\sqrt{\pi}} \int_{s} \vec{n} \cdot\left(\vec{e}_{r} \times \overrightarrow{h_{i}}\right) \exp [\mathrm{j} k \vec{r}(\vec{i}-\vec{s})] \mathrm{d} S
$$

where $\sqrt{\sigma_{p o}}$ is the RCS of a single facet, $\mathrm{j}$ is the imaginary unit, $\mathrm{j}^{2}=-1 . k=2 \pi / \lambda$ is the free space wavenumber, $\lambda$ is the radar incident wavelength, $S$ is the area of the aircraft radiated by the radar, $\vec{n}$ is the unit normal vector of the surface, $\vec{e}_{r}$ is the unit vector of the electromagnetic polarization direction of the receiving antenna, $\vec{h}_{i}$ is the unit vector of the field direction of the incident wave, the symbol "." represents the dot product and " $x$ " represents difference product, $\vec{r}$ is the vector from the local origin to the surface element $\mathrm{d} S, \vec{i}$ is the unit vector of the incident direction. $\vec{s}$ is the unit direction of the scattering direction.

The method of equivalent currents is expressed as[15]:

$$
\sqrt{\sigma_{\text {ecm }}}=\frac{1}{\sqrt{\pi} \sin \theta}\left[\left(\vec{E}_{o}^{i} \cdot \vec{t}\right) f \vec{s} \times(\vec{s} \times \vec{t})-Z_{0}\left(\vec{H}_{0}^{i} \cdot \vec{t}\right) g \vec{s} \times \vec{t}\right] \cdot e^{-j 2 k k_{i} \cdot \vec{s}} \frac{\sin (k \vec{k} \cdot \vec{s})}{k \vec{l} \cdot \vec{s}}
$$

where $\sqrt{\sigma_{e c m}}$ is the RCS of the single edge. $\vec{t}$ is the unit tangent vector of the edge. $\theta$ is the angle between the incident ray $\vec{i}$ and $\vec{t} . \vec{E}_{o}^{i}$ is the strength of the incident electric field. $f$ and $g$ are the Yoffie Rousseff diffraction coefficient. $Z_{0}$ is the wave impedance in vacuum in $\Omega . \vec{H}_{o}^{i}$ is the strength of the incident magnetic field. $\vec{r}_{t}$ is the position vector of the middle of the edge. $\vec{i}$ is the vector of the edge.

The RCS of the strike fighter is the sum of $n$ facets and $m$ edges, which can be expressed as:

$$
\sigma=\left|\sum_{i=1}^{n}\left(\sqrt{\sigma_{p o}}\right)_{i}+\sum_{j=1}^{m}\left(\sqrt{\sigma_{e c m}}\right)_{j}\right|^{2}
$$

where $\sigma$ is the RCS of the strike fighter

The arithmetic average RCS of the strike fighter is shown as below: 


$$
\bar{\sigma}_{n \sim N}^{\beta}=\frac{1}{N-n+1} \sum_{\varphi=n}^{N} \sigma_{\varphi}^{\beta}
$$

In the formula, $\sigma_{\varphi}^{\beta}$ is the RCS in square meters when the pitch angle of the incident wave is $\beta$ and the azimuth of the aircraft is $\varphi$.where $\bar{\sigma}_{n \sim N}^{\beta}$ is the average value of RCS when the pitch angle of the incident wave is $\beta$ and the azimuth of the aircraft $\varphi$ ranges from $\mathrm{n}$ to $\mathrm{N}$.

Convert the units of the RCS, we have

$$
\sigma_{d B s m}=10 \lg _{10} \sigma
$$

where $\sigma_{d B s m}$ is the RCS of the strike fighter in another unit dBsm.

\subsection{Experimental verification}

To verify the validity of the numerical simulation method to calculate the RCS of strike fighter based on the physical optics and MEC, an experiment has been performed, in which only the geometric shape of the aircraft is take into account while the effect of the stealth coating on the RCS of the aircraft is not considered.

The procedure of the experiment is:

(1) Build the 3D digital model of the aircraft in CATIA (see Fig. 1(a)).

(2) Use the 3D Printers to print the wax mold (see Fig.

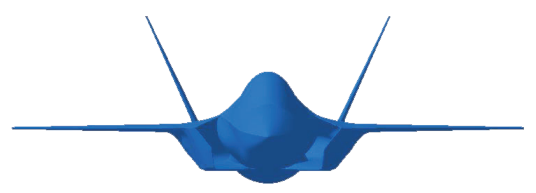

(a) 3D digital mode

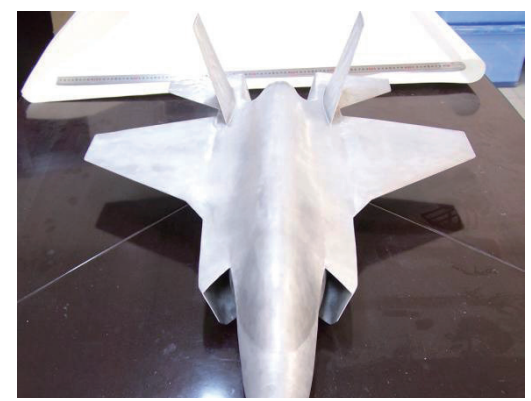

(c) Cast aluminum model $1(b))$.

(3) Build the sand mold cavity using the wax mold and then get the cast aluminum model from the mold cavity (see Fig. 1(c)).

(4) Conduct the electromagnetic test of the model in the anechoic chamber (see Fig. 1(d)).

(5) Compare the RCS feature curve from the electromagnetic test with that obtained by numerical simulation method (see Fig. 2).

The initial conditions of the numerical simulation and the

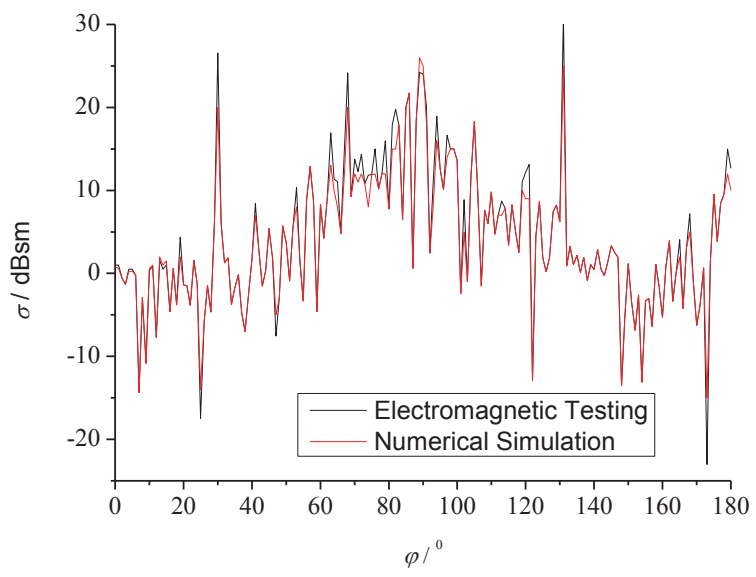

Fig. 2. RCS feature curve comparison between numerical simulation and experimental verification

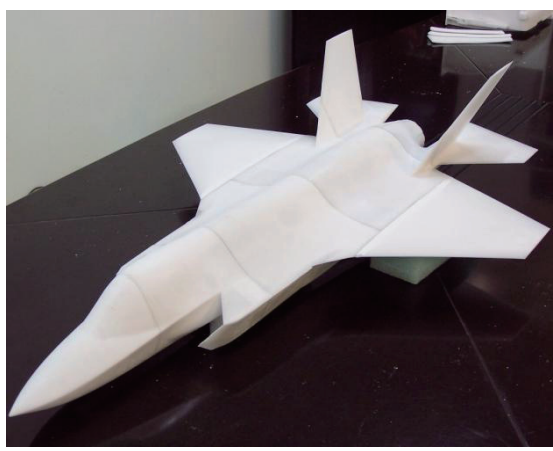

(b) Wax mold

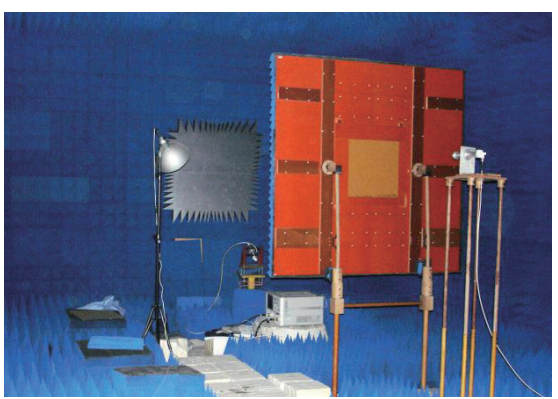

(d) Anechoic chamber

Fig. 1. Experimental verification 
experiment include: the radar incident wave is on X-band, the pitch angle of the wave is $0^{\circ}$, the horizontal polarization mode is chosen and the azimuth angle of the aircraft is $0 \sim 180^{\circ}$. The RCS feature curve of the 3D digital model in Fig. 1 (a) is obtained by the numerical simulation based on the physical optics and MEC(see Fig. 2). Another curve obtained by the electromagnetic test in the anechoic chamber is also shown in Fig. 2. The comparison analysis shows that the curve obtained from the numerical simulation method is in good agreement with the curve from the electromagnetic test.

The experiment has proved the accuracy of the numerical simulation method. Thus it can be applied to analyze the RCS feature of the three-surface conceptual aircraft.

\section{Conceptual design}

This paper concentrates on the conceptual design and the stealth design of the three-surface strike fighter, based on the design concept of the advanced fighter such as
Su-37, F-22, F-35, T-50 and so on. The conceptual aircraft is a heavy strike fighter with single seat, twin-engine and three-surface, involving several stealth technology:(1) The camber angles of double vertical tails and double ventral fins are both $35^{\circ}$ so that the side scattering RCS can be reduced. (2) The leading sweepbacks of the canard, wing and horizontal tail are all $45^{\circ}$ and their trailing sweepbacks are the same to reduce the crest amount of the RCS at the azimuth range $0^{\circ}$ to $360^{\circ}$. (3) The aircraft uses the internal weapon bays with air-to-air missiles or air-to-surface missiles to reduce the RCS when carrying missiles. (4) The S-shaped inlet, which can keep the radar wave from directly radiating the straightening vanes and the compressor blades, is used to reduce the RCS of the inlet cavity when the radar wave reflects repeatedly in the inlet. (5) The entrance of the inlet is the supersonic inlet with boundary layer diverter which can enlarge the internal weapon bays to carry the long-range air-to-surface missiles compared to the DSI inlet. (6) Continuous surface and blended wing body technology are adopted in designing fuselage to reduce the RCS of all the joints. (7) The integrative canopy

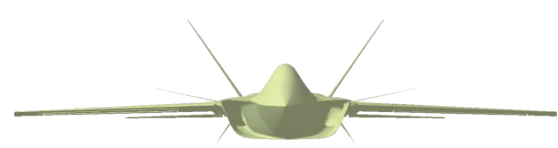

(a) Front view

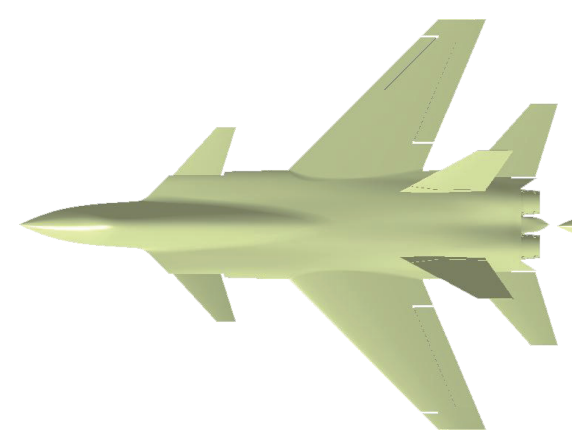

(c) Top view

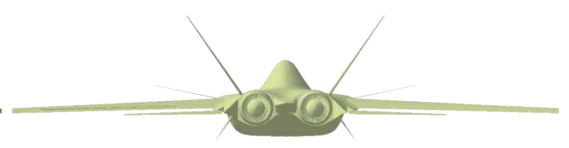

(b) Rear view

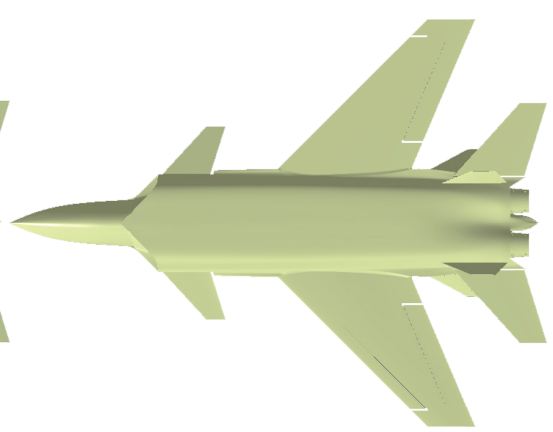

(d) Upward view

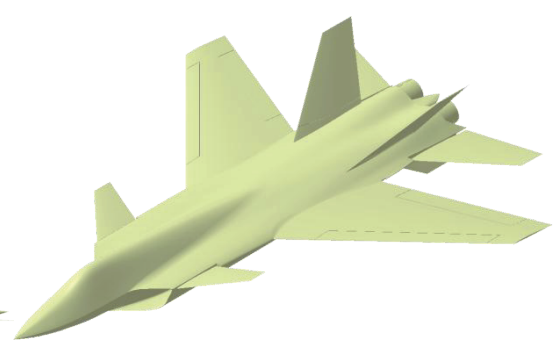

(f) Stereogram

(e) Left view

Fig. 3. 3D digital model of the conceptual design three-surface strike fighter 
based on the golden coating technology is in bubble shape, smoothly blended with the aircraft nose to reduce the RCS of the cockpit cavity and the canopy joint. (8) The engine is a thrust-vectoring motor with an exhaust nozzle exit optimized to reduce the infrared radiation. (9) The aircraft nose is designed with edges to reduce the forward and side scattering RCS.

The 3D digital model of the three-surface strike fighter designed by CATIA is shown in Fig. 3. The schemes of carrying missiles inside are shown as Fig. 4.

The schemes of carrying missiles are listed in details: (1) Scheme 1 is to carry 5 mid-range air-to-air missiles and 1 mid-range air-to-surface missile for air combat and ground attack. (2) Scheme 2 is to carry 10 mid-range air-to-air missiles for air combat. (3) Scheme 3 is to carry 1 long-range air-to-surface missile for ground attack.

The basic parameters of the conceptual design threesurface strike fighter are shown in Table 1:

The stealth properties of the digital model need to be analyzed to estimate the merits of the conceptual design.

Table 5. Basic parameters of the conceptual design three-surface strike fighter

\begin{tabular}{cc}
\hline Parameters & Dimension \\
\hline Aircraft length & $21.949 \mathrm{~m}$ \\
Aircraft height & $5.82 \mathrm{~m}$ \\
Span & $16.742 \mathrm{~m}$ \\
Leading sweepback & $45^{\circ}$ \\
Trailing sweepback & $23^{\circ}$ \\
Tip chord & $1.915 \mathrm{~m}$ \\
Blended-wing chord & $6.718 \mathrm{~m}$ \\
Number of engines & 2 \\
Canard airfoil profile & NACA64a202 \\
Wing airfoil profile & NACA64a204 \\
Horizontal tail airfoil profile & NACA64a202 \\
Vertical tail airfoil profile & NACA64a003 \\
\hline
\end{tabular}

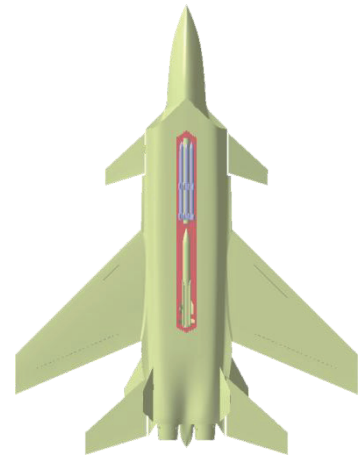

(a) Scheme 1

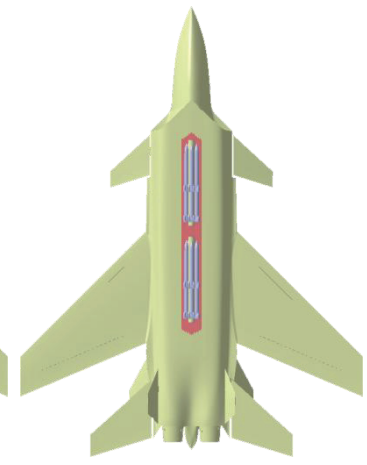

(b) Scheme 2

\section{Numerical simulation of RCS properties of the strike fighter}

The analysis of the RCS properties includes three parts: the RCS feature analysis of the concept three-surface strike fighter, the RCS feature analysis of the fighter with missiles and the comparison analysis with other stealth aircrafts.

\subsection{RCS property analysis of the strike fighter}

This section is focus on the RCS property analysis of the strike fighter, using equation (1) (3) based on the physical optics and MEC with RCSPlus. The RCS feature curve obtained by numerical simulation and the RCS scattering intensity feature of the strike fighter is given in Fig. 5 and Fig. 6 respectively. The RCS mean value feature is shown in table 2 . In the process of numerical simulation, the model of the fighter is slightly simplified for convenience without influencing the precision. A slope is used to close the inlet along with the boundary layer diverter and a conical surface is used to close the nozzle of the afterburner.

As Fig. 5 shows, on condition that the incident wave is $\mathrm{X}$-band, the pitch angle is $0^{\circ}$ and the azimuth ranges from $0^{\circ}$ to $360^{\circ}$, the RCS feature of the strike fighter is : (1) The forward scattering RCS mean value is $-2.178 \mathrm{dBsm}$ at a range of $\pm 30^{\circ}$. (2) The side scattering RCS mean value is $14.890 \mathrm{dBsm}$. (3) The back scattering RCS mean value is $6.909 \mathrm{dBsm}$ at a range of $\pm 30^{\circ}$.

Some other conclusions can also be drawn from the curves in Fig. 5: (1) When the azimuth is $45^{\circ}$ or $315^{\circ}$, the peak of the RCS is higher because the same leading sweepback of the canard, wing and vertical tails leads to the RCS superposition. (2) When the azimuth is $90^{\circ}$ or $270^{\circ}$, the peak of the RCS is higher because of the higher side scattering RCS value of the fuselage. (3) When the azimuth ranges from $-10^{\circ}$ to $+10^{\circ}$, the RCS is lower because of the aircraft nose with

Fig. 4. Schemes to carry missiles

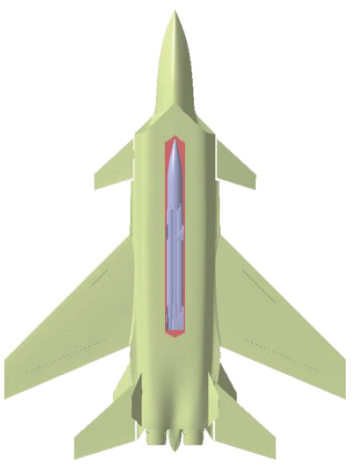

(c) Scheme 3 
edges and the S-shaped inlet.

Under the condition that the incident wave is X-band, the pitch angle is $0^{\circ}$ and the azimuth ranges from $0^{\circ}$ to $360^{\circ}$, altogether 360 RCS scattering intensity images are obtained in the numerical simulation. Some representative images are shown as Fig. 6.

Figure 6 shows some RCS properties as below: (1) The forward scattering RCS of the radome, the canopy and the inlet entrance is higher, so these parts need to be optimized. (2) The side scattering RCS of the fuselage is higher, but the RCS of double vertical tails and ventral fins is lower because the camber angle reduces the side scattering RCS. So the fuselage needs to be optimized. (3) As the main part of the infrared radiation scattering and the RCS scattering, the back

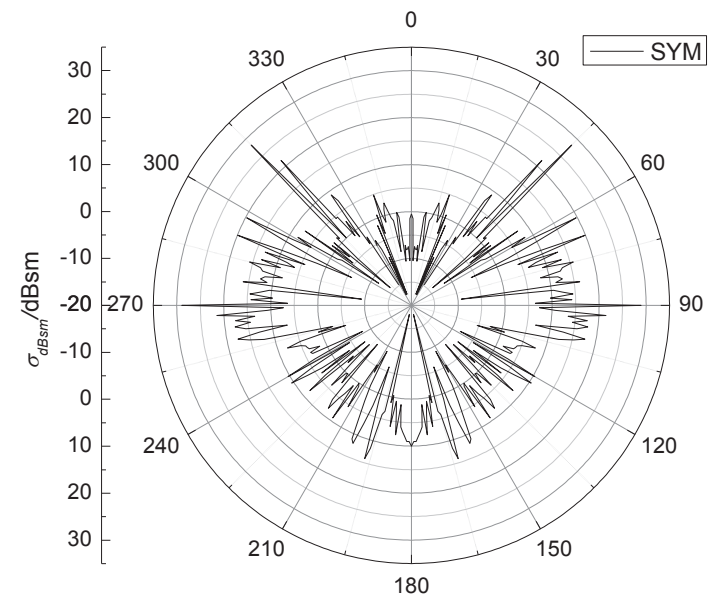

Fig. 5. RCS feature curve of the three-surface strike fighter on X-band scattering RCS of the engines is higher. So the engines need to be optimized too.

This paper also performs an omnidirectional numerical simulation besides the RCS property on X-band.

The initial conditions include: The radar bands are X-band, S-band and UHF-band, the pitch angle of the incident wave ranges from $-15^{\circ}$ to $+15^{\circ}$, the azimuth ranges from $0^{\circ}$ to $360^{\circ}$ and the polarizations of radar are horizontal polarization and vertical polarization. The consequences show as below: The numerical simulation concludes $7 \times 3 \times 2=42$ RCS curves

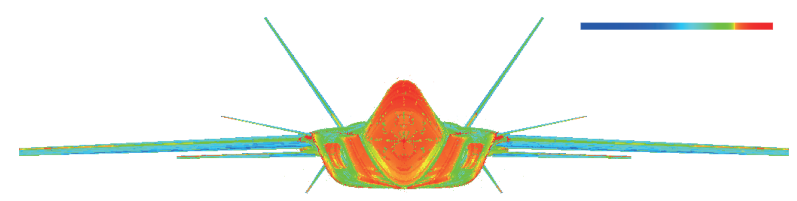

(a) Front view

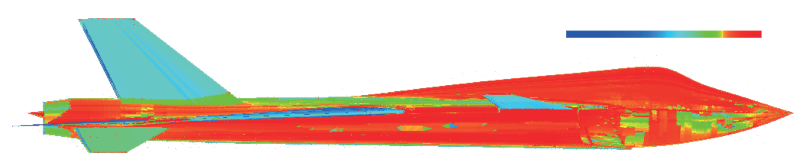

(b) Left view

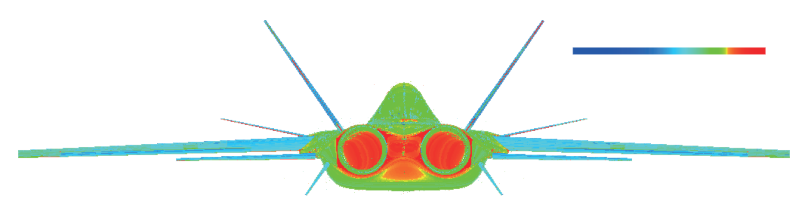

(c) Rear view

Fig. 6. RCS scattering intensity feature of the strike fighter

Table 2. RCS mean value feature of the three-surface strike fighter

\begin{tabular}{|c|c|c|c|c|c|c|c|}
\hline \multirow{3}{*}{ Radar band } & \multirow{3}{*}{ Pitch angle } & \multicolumn{6}{|c|}{ RCS mean value at the azimuth range from $-30^{\circ}$ to $+30^{\circ}$} \\
\hline & & \multicolumn{3}{|c|}{ H-H polarization } & \multicolumn{3}{|c|}{ V-V polarization } \\
\hline & & Forward & Side & Back & Forward & Side & Back \\
\hline $\mathrm{X}$ & $15^{\circ}$ & -3.393 & 13.570 & 4.625 & -3.354 & 13.571 & 4.876 \\
\hline $\mathrm{X}$ & $10^{\circ}$ & -2.797 & 11.754 & 5.265 & -2.732 & 11.765 & 5.263 \\
\hline $\mathrm{X}$ & $5^{\circ}$ & -6.359 & 10.366 & 4.890 & -6.228 & 10.284 & 5.281 \\
\hline $\mathrm{X}$ & $0^{\circ}$ & -2.178 & 14.890 & 6.909 & -2.177 & 14.898 & 8.614 \\
\hline$X$ & $-15^{\circ}$ & -2.477 & 13.489 & 9.238 & -2.512 & 13.538 & 9.475 \\
\hline S & $15^{\circ}$ & -2.618 & 11.718 & 1.470 & -2.152 & 11.808 & 1.637 \\
\hline $\mathrm{S}$ & $10^{\circ}$ & -2.766 & 10.453 & 2.993 & -2.359 & 10.553 & 3.505 \\
\hline S & $5^{\circ}$ & -3.178 & 10.635 & 4.238 & -2.765 & 10.602 & 4.740 \\
\hline S & $0^{\circ}$ & -1.554 & 10.277 & 7.959 & -0.868 & 10.290 & 8.231 \\
\hline UHF & $10^{\circ}$ & -0.402 & 8.141 & 2.883 & 1.470 & 8.552 & 6.390 \\
\hline UHF & $5^{\circ}$ & -0.362 & 8.003 & 2.336 & -0.420 & 7.339 & 5.655 \\
\hline UHF & $0^{\circ}$ & 0.640 & 7.530 & 2.803 & 1.053 & 6.473 & 5.831 \\
\hline UHF & $-5^{\circ}$ & 0.843 & 7.391 & 3.076 & 1.601 & 7.468 & 4.984 \\
\hline UHF & $-10^{\circ}$ & 0.346 & 8.620 & 2.954 & 1.512 & 9.500 & 4.090 \\
\hline UHF & $-15^{\circ}$ & 1.485 & 11.836 & 3.722 & 2.042 & 12.330 & 5.144 \\
\hline
\end{tabular}


of the strike fighter and $7 \times 3 \times 2 \times 360=15120$ RCS scattering intensity images in total. The RCS mean value features of the strike fighter are obtained after calculating the arithmetic average and converting units based formula (4) and (5) (see Table 2).

From Table 2 the RCS mean value features of the strike fighter show: (1) Supposing that the radar band is X-band, the polarization is $\mathrm{H}-\mathrm{H}$ polarization, and the forward azimuth ranges from $-30^{\circ}$ to $+30^{\circ}$, the arithmetic mean of forward scattering RCS $\bar{\sigma}_{\mathrm{dBsm}}^{ \pm 30^{\circ}} \ngtr-0.782 \mathrm{dBsm}$, the arithmetic mean of side scattering RCS at the side azimuth range of $\pm 30^{\circ}$ is $\bar{\sigma}_{\mathrm{dBsm}}^{ \pm 30^{\circ}} \ngtr 14.890 \mathrm{dBsm}$ and the back scattering RCS arithmetic mean at the back azimuth range of $\pm 30^{\circ}$ is $\bar{\sigma}_{\mathrm{dBsm}}^{ \pm 30^{\circ}} \ngtr 9.657 \mathrm{dBsm}$. (2) Under the condition that the radar band is X-band, the polarization is V-V polarization, the forward scattering RCS arithmetic mean at the forward azimuth range of $\pm 30^{\circ}$ is $\bar{\sigma}_{\mathrm{dBsm}}^{ \pm 30^{\circ}} \ngtr-0.517 \mathrm{dBsm}$, the side scattering RCS arithmetic mean at the side azimuth range of $\pm 30^{\circ}$ is $\bar{\sigma}_{\mathrm{dBsm}}^{ \pm 30^{\circ}} \ngtr 14.898 \mathrm{dBsm}$ and the back scattering RCS arithmetic mean at the back azimuth range of $\pm 30^{\circ}$ is $\bar{\sigma}_{\mathrm{dBsm}}^{+30^{\circ}} \ngtr 10.036 \mathrm{dBsm}$. Similarly, the RCS mean value features of the strike fighter are also analyzed under the condition of X-band, UHF-band, $\mathrm{H}-\mathrm{H}$ polarization and $\mathrm{V}-\mathrm{V}$ polarization.

The following work is about the RCS feature comparison analysis with other typical three-surface aircrafts.

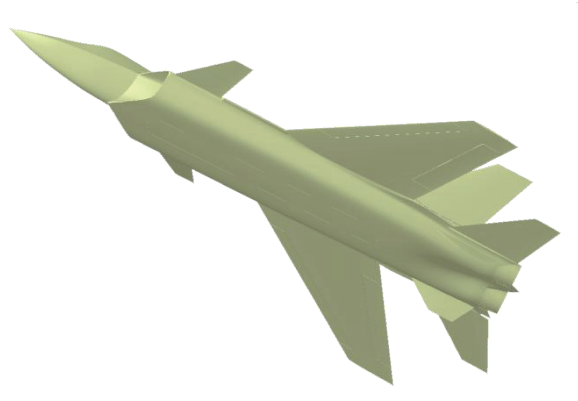

(a) Model A

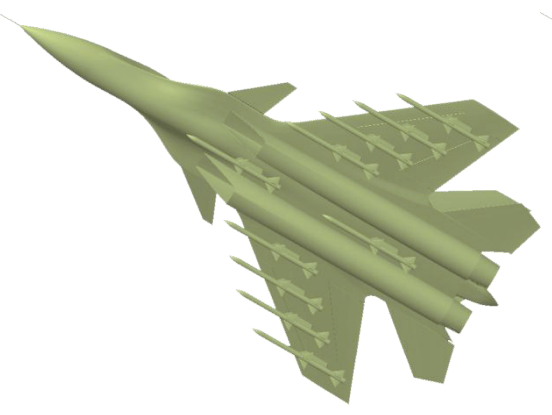

(c) Scheme 2 of model B

\subsection{RCS feature analysis of the typical three-surface aircraft with missiles}

The Russian aircrafts Su-33 and Su-37 are typical threesurface aircrafts. This section is focus on the RCS comparison analysis between the conceptual design aircraft and the Su37 with missiles.

The conceptual design three-surface strike fighter is shown in Fig. 7(a). Its 3d digital mock-up is shown in Fig. 3 and the schemes to carry missiles are shown in Fig. 4.

The schemes of Su-37 to carry missiles are shown in Fig. 7(b) (d). The digital mock-up of Su-37 is short for B. In order to compare with the conceptual design strike fighter, the schemes to carry missiles are designed as below: (1) In scheme 1 , the aircraft carries 5 mid-range air-to-air missiles and 1 mid-range air-to-surface missile outside (see Fig. 7(b)). In scheme 2, the aircraft carries 10 mid-range air-toair missiles outside (see Fig. 7(c)). In scheme 3, the aircraft carries 1 long-range air-to-surface missile outside (see Fig. $7(d))$.

Analyze the three schemes of model A and model B to compare the RCS feature based on the physical optics and MEC with the RCSPlus software. The consequences of the numerical simulation include: (1) The RCS feature curve of the typical three-surface aircraft system is obtained (see Fig. 8). (2) The RCS scattering intensity feature of the typical

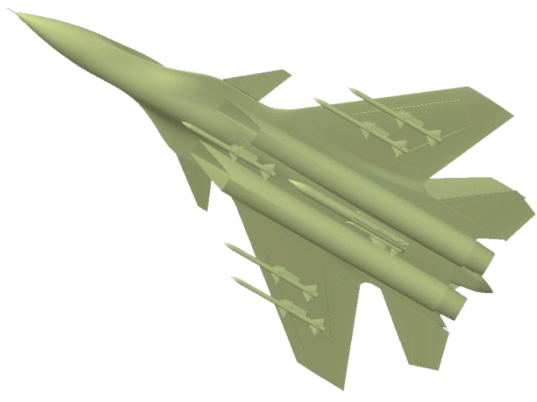

(b) Scheme 1 of model B

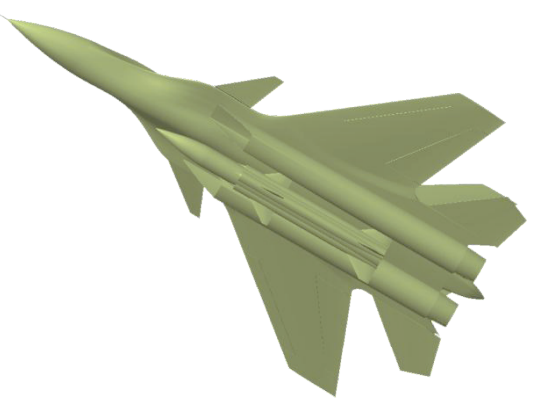

(d) Scheme 3 of model B

Fig. 7. Typical three-surface aircraft system 
three-surface aircraft system is obtained (see Fig. 9). (3) The RCS mean value comparison of the typical three-surface aircraft system is obtained (see Table 3 ).

From Fig. 8, when the radar band is X-band, the pitch angle is $0^{\circ}$, the polarization is horizontal polarization, calculate the arithmetic mean and transform the units with the formula (4) and (5), the RCS feature of the typical threesurface aircraft system is shown as below: (1) For model A, the forward scattering RCS arithmetic mean at the forward azimuth range of $\pm 30^{\circ}$ is $-2.178 \mathrm{dBsm}$, the side scattering RCS arithmetic mean at the side azimuth range of $\pm 30^{\circ}$ is $14.890 \mathrm{dBsm}$ and the back scattering RCS arithmetic mean at the back azimuth range of $\pm 30^{\circ}$ is $6.909 \mathrm{dBsm}$. (2)Similarly, the RCS mean value features of three schemes of model B are also analyzed. And the consequences are summarized in table 3 .

This paper also research on the RCS feature of different parts. On the condition that the radar band is X-band, the polarization is horizontal polarization and the pitch angle of the incident wave is $5^{\circ}, 0^{\circ}$ and $-5^{\circ}, 12$ RCS feature curves and

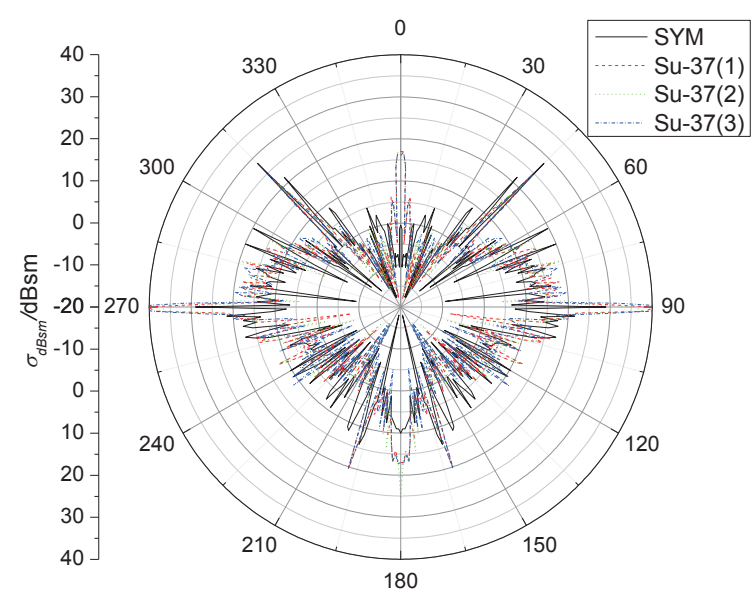

Fig. 8. RCS feature curve of the typical three-surface aircraft system
4320 RCS scattering intensity images are obtained after the numerical simulation.

Choose the RCS scattering intensity images to do research, such as the images obtained on the condition that the pitch angle of the incident wave is $-5^{\circ}$ and the azimuth is 10 (see in Fig. 9). The consequences show that the RCS scattering intensity of the three schemes of model B is high because the scattering intensity of the missiles outside increases the scattering intensity of the aircraft.

And then 12 RCS feature curves are analyzed to obtain the RCS mean value comparison of the typical three-surface aircraft through mathematical statistics and unit conversion (see Table 3).

On the condition that the radar band is X-band and the pitch angle is $0^{\circ}$, the consequences show: (1) Compared with scheme 1 of model B, the forward RCS mean value of model A at the forward azimuth range of $\pm 30^{\circ}$ is reduced to $14.9 \%$, the side RCS mean value at the side azimuth range of $\pm 30^{\circ}$ is reduced to $9.6 \%$, and the back RCS mean value at the back azimuth range of $\pm 30^{\circ}$ is reduced to $40.2 \%$. (2) Compared

Table 3. RCS mean value comparison of typical three-surface aircraft on $\mathrm{X}$-band and $\mathrm{H}-\mathrm{H}$ polarization

\begin{tabular}{ccccc}
\hline \multirow{2}{*}{ scheme } & \multirow{2}{*}{ Pitch angle } & \multicolumn{3}{c}{ RCS mean value at the azimuth range of $\pm 30^{\circ}$} \\
\cline { 3 - 5 } & 5 & -6.359 & 10.366 & 4.890 \\
\hline \multirow{2}{*}{ Model A } & 0 & -2.178 & 14.890 & 6.909 \\
& -5 & -2.315 & 13.445 & 9.657 \\
& 5 & -2.348 & 17.373 & 4.995 \\
Scheme 1 of model B & 0 & 6.077 & 25.066 & 10.864 \\
& -5 & -0.451 & 22.300 & 4.064 \\
Scheme 2 of model B & 5 & -1.914 & 15.634 & 4.920 \\
& 0 & 6.161 & 22.751 & 13.609 \\
Scheme 3 of model C & -5 & 0.139 & 17.928 & 3.733 \\
& 5 & -1.826 & 18.182 & 3.486 \\
& 0 & 6.473 & 29.604 & 10.798 \\
& -5 & -1.621 & 26.764 & 2.326 \\
\hline
\end{tabular}

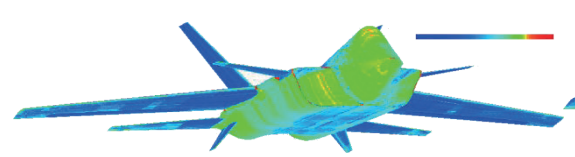

(a) Model A

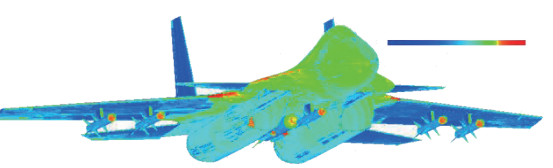

(b) Scheme 1 of model B

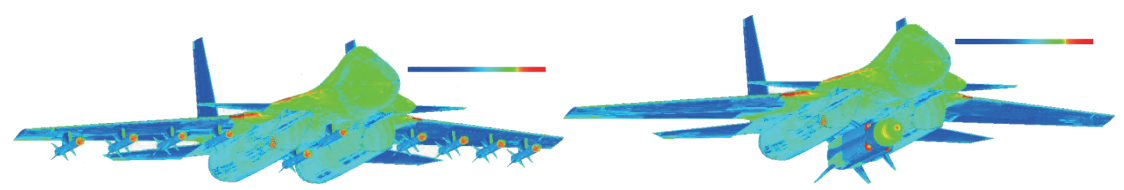

(c) Scheme 2 of model B

(d) Scheme 3 of model B

Fig. 9. RCS scattering intensity feature of typical three-surface aircraft system (pitch angle $-5^{\circ}$, azimuth $10^{\circ}$ ) 


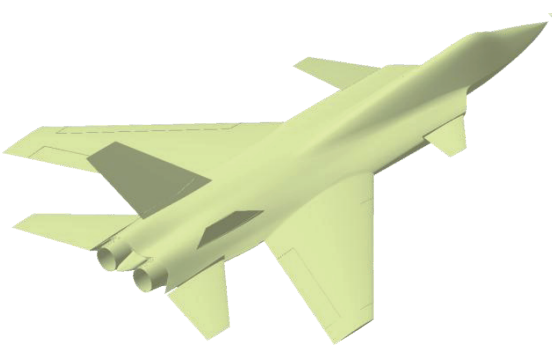

(a) Model A

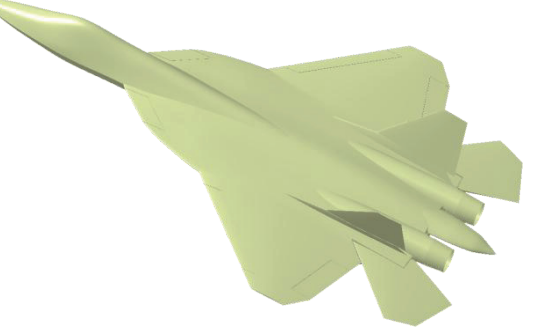

(b) Model C

Fig. 10. Typical stealth aircraft

with scheme 2 of model B, the forward RCS mean value is reduced to $14.6 \%$, the side RCS mean value is reduced to $16.36 \%$ and the back RCS mean value is reduced to $21.37 \%$.

(3) Compared with scheme 3 of model B, the forward RCS mean value is reduced to $13.6 \%$, the side RCS mean value is reduced to $3.3 \%$ and the back RCS mean value is reduced to $40.8 \%$.

Above all, the stealth feature of the conceptual threesurface strike fighter with missiles inside is better than the ones with missiles outside.

\subsection{Comparison analysis with other stealth aircrafts}

The following analysis is about the RCS comparison analysis between the conceptual three-surface strike fighter and T-50 stealth aircraft.

Based on the Russian aircraft T-50, the 3D digital mock-up of T-50 which is short for model C is designed in CATIA (see Fig. 10(b)).

When the radar band is X-band and the polarization is horizontal polarization, the numerical simulation consequences of model A and model C include the RCS

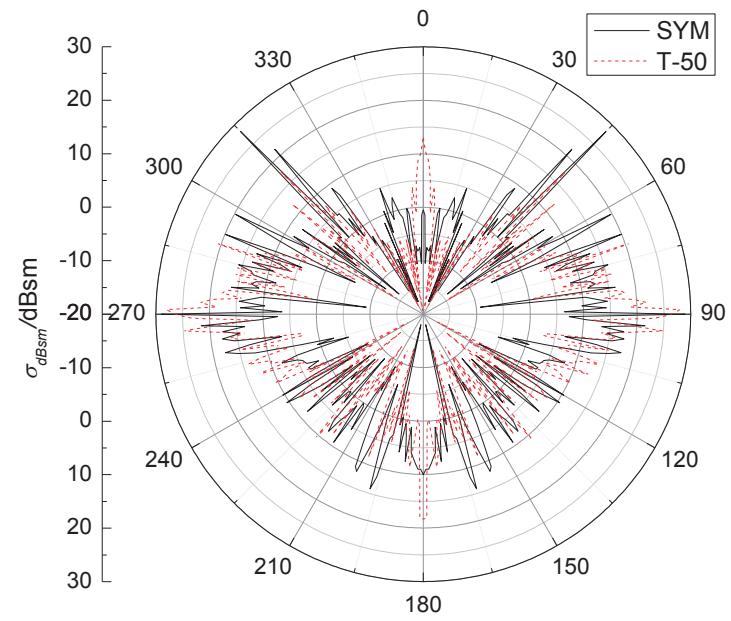

Fig. 11. RCS feature curves of the typical stealth aircraft feature curves (see Fig. 11), the RCS scattering intensity feature (see Fig. 12) and the RCS mean value comparison (see Table 4).

From Fig. 11, when the radar band is X-band, the pitch angle of the incident wave is $0^{\circ}$ and the polarization is horizontal polarization, after mean value calculation and unit conversion using formula (4) and (5), the RCS features of Model C show: the forward scattering RCS arithmetic mean at the forward azimuth range of $\pm 30^{\circ}$ is $1.954 \mathrm{dBsm}$, the side scattering RCS arithmetic mean at the side azimuth range of $\pm 30^{\circ}$ is $16.612 \mathrm{dBsm}$ and the back scattering RCS arithmetic mean at the back azimuth range of $\pm 30^{\circ}$ is $7.587 \mathrm{dBsm}$.

From Table 4, when the pitch angle is $0^{\circ}$, the forward RCS of model C is higher than model A because the radar wave can directly radiate to the engine compressor blades of model C so that the forward RCS increases (see Fig. 12(c) and Fig. 6(a)).

From table 4 , on the condition that the radar band is $\mathrm{X}$-band and the pitch angle is $0^{\circ}$, compared with model $\mathrm{C}$, the

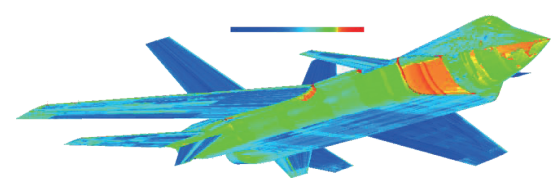

(a) Model A, pitch angle $-10^{\circ}$, azimuth $30^{\circ}$

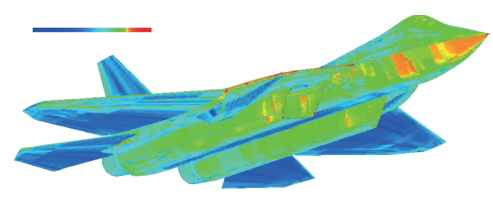

(b) Model C, pitch angle $-10^{\circ}$, azimuth $30^{\circ}$

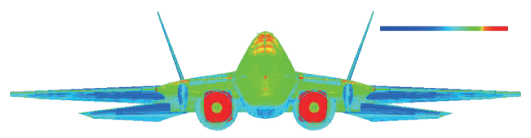

(c) Model $C$, pitch angle $0^{\circ}$, azimuth $0^{\circ}$

Fig. 12. RCS scattering intensity feature of typical stealth aircraft 
Table 4. RSC feature comparison on $\mathrm{X}$-Band and $\mathrm{H}-\mathrm{H}$ polarization

\begin{tabular}{lcccc}
\hline \multirow{2}{*}{ Scheme } & \multirow{2}{*}{ Pitch angle } & \multicolumn{3}{c}{ RCS mean value at the azimuth range of $\pm 30^{\circ}$} \\
\cline { 2 - 5 } & & Forward & Side & Back \\
\hline \multirow{3}{*}{ Model A } & 10 & -2.797 & 11.754 & 5.265 \\
& -10 & -2.178 & 14.890 & 6.909 \\
& 10 & -0.782 & 13.380 & 2.007 \\
Model C & 0 & -5.949 & 12.286 & 5.246 \\
& -10 & 1.954 & 16.612 & 7.587 \\
& -2.100 & 19.177 & 3.927 \\
\hline
\end{tabular}

forward RCS mean value of model A at the forward azimuth range of $\pm 30^{\circ}$ is reduced to $38.61 \%$, the side RCS mean value at the side azimuth range of $\pm 30^{\circ}$ is reduced to $67.26 \%$, and the back RCS mean value at the back azimuth range of $\pm 30^{\circ}$ is reduced to $85.54 \%$.

Above all, the stealth property of the conceptual threesurface strike fighter doesn't appear to be much different from the T-50. They are on the same order of magnitude. But at most azimuth, the RCS of the conceptual fighter is lower than T-50. On the other hand, the conceptual fighter has a more optimized scheme to carry missiles because it can carry long-range air-to-surface missiles.

\section{5. conclusion}

This paper concentrates on the conceptual design of three-surface strike fighter. Based on the physical optics and MEC, a numerical simulation of the RCS feature is carried out. Through the numerical simulation and the comparison analysis, the conclusions are given as below:

(1) The numerical simulation method to calculate the RCS feature based on the physical optics and MEC is proved to be scientific and accurate after comparison with the electromagnetic test.

(2) The conceptual three-surface strike fighter has stealth property and the ability of air combat or air-to-ground combat.

(3) Compared with the digital mock-up of the Su-37 with missiles outside, the RCS of the conceptual three-surface strike fighter with missiles inside is lower. The conceptual fighter has better stealth ability.

(4) The order of magnitude of the stealth property of the conceptual three-surface strike fighter is the same as T-50. But at most azimuth, the RCS of the conceptual fighter is lower than T-50 because of the insufficient design of the inlet of T-50. Moreover the conceptual fighter has a more optimized ability of air-to-ground combat because it can carry long-range air-to-surface missiles.

This paper centers on the conceptual design and the RCS numerical simulation of three-surface strike fighter. And it is expected to provide reference and technical support for conceptual design and stealth design of the military aircraft.

\section{References}

[1] Suresh, R. P., and Christopher, L. B., "F/A-22 Vertical Tail Buffet Strength Certification," AIAA 2005-2292.

[2] Paul, M. B., "Genesis of the F-35 Joint Strike Fighter," Journal of Aircraft, Vol.46, No.6, 2009, pp.1825-1836.

[3] Cong, S., and Peng, Z., "Lo Requirements and Solutiona of Avionics/RF System for Advanced Aircraft," Acta Aeronautica et Astronautica Sinica, Vol.29, No.6, 2008, pp.1472-1481.

[4] Peilin, H., and Zhanhe, L., "Research on Electromagnetic Scattering Characteristics of Slits on Aircraft," Acta Aeronautica et Astronautica Sinica, Vol.29, No.3, 2008, pp.675-680.

[5] Shen Shou Max Chung, "FDTD simulations on radar cross sections of metal cone and plasma covered metal cone," Vacuum, Vol.86, No.7, 2012, pp.970-984.

[6] Minjie, H., Mingyun, L., and Jun, H., "Recognition of the major scattering sources on complex targets based on the high frequency radar cross section integrated calculation technique," Journal of Shanghai University, Vol.13, No.4, 2009, pp.115-121.

[7] Ledger, P. D., and Morgan, K., "An Adjoint Enhanced Reduced-Order Model for Monostatic RCS Computation," Electromagnetics, Vol.28, No.1-2, 2008, pp.54-76.

[8] JInzu, J., Peilin, H., and Bo, L., "Study on singularity of physical theory of diffraction," Systems Engineering and Electronics, Vol.34, No.10, 2012, pp.1987-1993.

[9] Jinzu, J., Daqing, H., and Peilin, H., “Connective boundary's electromagnetic leakge in finite-difference timedomain," Journal of Beijing University of Aeronautics and Astronautics, Vol.39, No.2, 2013, pp.159-163.

[10] Huanyu, L., Peng, B., and Xuhua, W., "Analysis of influence of radio frequency on RF stealth," Systems Engineering and Electronics, Vol.34,No. 6, 2012, pp.1108-1112.

[11] Zhu Xijuan, Eriqitai, and Li Jiajun. "Effect of jet mixing enhancement by pulsed blowing on infrared radiation of plume," Journal of Beijing University of Aeronautics and Astronautics, Vol.37, No.5, 2011, pp.551-555.

[12] Kuizhi, Y., Cong, S., and Hu, Liu., "Numerical simulation on the RCS of combat aircraft for mounted missile," Systems Engineering and Electronics, Vol.36, No.1, 2014, pp.62-67.

[13] Kuizhi, Yue., Cong, S., and Jinzu, Ji., "Numerical simulation on the stealth characteristics of twin-vertical-tails 
for fighter," Journal of Beijing University of Aeronautics and Astronautics, Vol.40, No.2, 2014, pp.160-165.

[14] Baussard, A., Rochdi, M., and Khenchaf, A., "PO/ MEC-based bistatic scattering model for complex objects over a sea surface," Progress In Electromagnetics Research
(PIER), Vol.111, 2011, pp.229-251.

[15] Rochdi, M., Baussard, A., and Khenchaf, A., "Po/ MEC-Based Bistatic Scattering Model for Complex Objects Over a Sea Surface," IEEE International Radar Conference 2010, May 10-14, 2010, Washington DC, USA. 